\title{
Stable Rules for Electing Committees and Divergence on Outcomes
}

\author{
Eric Kamwa*† \\ Forthcoming in Group Decision and Negotiation
}

\begin{abstract}
For three-candidate elections, this paper focuses on the relationships that exist between three stable rules for committee elections and the classical rules from which each of these stable rules are adapted. When selecting committees, a voting rule is said to be stable if it always elects a fixed-size subset of candidates such that there is no candidate in this set that is majority dominated by a candidate outside (Barberà and Coelho, 2008; Coelho, 2004). There are some cases where a committee selected by a stable rule may differ from the committee made by the best candidates of the corresponding classical rule from which this stable rule is adapted. We call this the divergence on outcomes. We characterize all the voting situations under which this event is likely to occur. We also evaluate the likelihood of this event using the impartial anonymous culture assumption. As a consequence of our analysis, we highlight a strong connection between three Condorcet consistent rules: the Dodgson rule, the Maximin rule and the Young rule.
\end{abstract}

Keywords : Committee; Condorcet; Stable rule; Maximin; Dodgson; Young; Divergence on outcomes; Probability.

\footnotetext{
${ }^{*}$ Université des Antilles. (凶): eric.kamwa@unicaen.fr

${ }^{\dagger}$ The paper was completed while the author was at Center for Research in Economics and Management, CREM UMR CNRS 6211 and Université du Havre, Faculté des Affaires Internationales.
} 


\section{Introduction}

The Condorcet committee à la Gehrlein which is a fixed-size subset of candidates such that each member majority dominates ${ }^{1}$ any outside contender was suggested by Gehrlein (1985) as a solution concept $^{2}$ for committee elections in respect with the Condorcet criterion ${ }^{3}$ (Condorcet, 1785). For fixed-size of committees, the Condorcet committee à la Gehrlein does not always exists. But there is a weak version of the Condorcet committee à la Gehrlein that is more likely to exist : a fixed-size subset of candidates such that none of its members is majority dominated by any outside contender. This is called the weak Condorcet committee à la Gehrlein (CCG, herefater). It is obvious that a Condorcet committee à la Gehrlein is also a CCG but the reverse is not true. For a given voting situation, there may exist more than one CCG while this is not the case for the Condorcet committee à la Gehrlein with an even number of voters ${ }^{4}$.

When a voting rule always selects a CCG when it exists, this rule is said to be stable (Barberà and Coelho (2008); Coelho (2004)). Coelho (2004) showed that almost all the classical voting rules are not stable. Up to our knowledge there are only six stables rules that have been suggested or identified in the social choice literature. These rules are the following ${ }^{5}$ :

- The Kemeny-Ratliff rule(KR) (Ratliff, 2003): When electing a committee of size $g(g \geq 2)$, this rule selects the subset(s) of $g$ candidates with the smallest total margin of loss in pairwise comparisons versus the $m-g$ remaining candidates. This rule is clearly an adaptation of the (Kemeny, 1959) rule ${ }^{6}$.

- The Dodgson-Ratliff (DR) (Ratliff, 2003): it selects the subset of $g$ candidates that requires the fewest number of adjacency switches to make this subset become a CCG. This rule is adapted from the (Dodgson, 1876) rule ${ }^{7}$.

- The Minimal Number of External Defeats Rule (NED) (Coelho, 2004): selects the committee(s) of size $g$ with the minimum total number of pairwise comparisons lost by its members versus the outside contenders.

- The Minimal size of External opposition Rule (SEO) (Coelho, 2004): given the highest margin of loss of a candidate in a committee against an outside candidate, this rule selects the

\footnotetext{
${ }^{1}$ A candidate $x$ majority dominates a candidate $y$ if he is preferred by more than half of the electorate to $y$.

${ }^{2}$ Notice that the concept of Condorcet committee $\grave{a}$ la Gehrlein is less restrictive than the one suggested by Schwartz (1986). The Schwartz set is the union of all Schwartz set components. A Schwartz set component is any non-empty set $S$ of candidates such that i) every candidate inside the set $S$ is pairwise unbeaten by every candidate outside $S$ and $i$ i) there is no non-empty proper subset of $S$ such that $i$ ) is fulfilled.

${ }^{3}$ The Condorcet criterion requires that when there is a Condorcet winner, this candidate must be chosen as the winner. A Condorcet winner is a candidate that beats each of the other candidates in pairwise comparisons.

${ }^{4}$ The uniqueness of the Condorcet committee à la Gehrlein is guaranteed only with an odd number of voters. For more on the relations between the Condorcet committee à la Gehrlein and the related concepts, see Miller (1980); Kamwa and Merlin (2013).

${ }^{5}$ Kamwa (2015b) have evaluated all the these stable rules on the basis of some appealing properties of voting rules.

${ }^{6}$ The Kemeny rule operates by computing distances from a given linear order to all the linear orders of a preferences profile. The Kemeny ranking is the linear order that minimizes this distance the total distance to the whole profile and the Kemeny winner is the candidate at the top of this ranking.

${ }^{7}$ In one-winner elections, the Dodgson rule elects the candidate who needs the fewest number of adjacency switches to win all his pairwise contests i.e to become the Condorcet winner.
} 
committee(s) with the smallest "highest margin of loss". As noticed by Coelho (2004), this rule is clearly an adaptation of the Maximin rule $^{8}$ to committee elections.

- The Young-Condorcet rule (YC) (Kamwa, 2015b): it selects the set of $g$ candidates that need the fewest number of deletions of voters to become a CCG. This rule is adapted from the Young rule ${ }^{9}$.

- The Minimal deletion of candidates rule (MDC) (Kamwa, 2015b): selects the set of $g$ candidates that need the fewest number of deletion of candidates to become a CCG.

As already mentioned, the KR is derived from the Kemeny rule, the DR from the Dodgson rule, the SEO from the Maximin rule and the YC from the Young rule. The Kemeny rule, the Dodgson rule, the Maximin rule and the Young rule are well known as Condorcet consistent rules since they always elect the Condorcet winner when he exists. Then, in this paper, will use the term "classical rules" to refer to these rules from which the stable rules are derived. Notice that if we want to select one-member committees, each of these stable rules is equivalent to the classical rule it is derived from. So, in this paper, our main concern will be on committees of at least two members. It is obvious that when there is a CCG, the stable rules will all select this committee. Coelho (2004) and Kamwa (2015b) focused on the relationships that can exist between the outcome sets of the above stable rules. They concluded that when there is no CCG, the stable rules may lead to disjoint outcome sets. Kamwa (2015b) showed that it is only in three-candidate elections that the outcome sets of the stable rule always have at least one element in common; more, he showed that in three-candidate a elections with no CCG, the KR, the DR, the SEO and the YC always select the same committee of two members while the NED and the MDC select all the committees of size two. Kamwa (2015b), Coelho (2004) and Ratliff (2003) showed that in general, each of the stable rules does not always select committees made of the top candidates (of the collective ranking) of the corresponding classical rule. We call this the "divergence on outcomes".

Ratliff (2003) described the divergence between the KR and the Kemeny rule in a sleek manner; he concluded that the KR and the Kemeny rule do not diverge when we select a two-member committee out of four candidates. For his own, Kamwa (2013) showed that it is also the case when selecting a two-member committees out of three candidates. In this paper, we will focus on the divergence between the DR and the Dodgson rule, between the SEO and the Maximin rule and between the $\mathrm{YC}$ and the Young rule. Notice that determining the collective ranking of the Dodgson rule/the Maximin rule/the Young rule can be an arduous task due to the complexity of the computation of the scores (Bartholdi et al., 1989; Betzler et al., 2008; Caragiannis et al., 1998; Hemaspaandra et al., 1997). For this reason, our analysis will be restricted to voting situations with three candidates. We assume two-member committee elections and we characterize all the voting situations under which one can get a divergence on outcomes. Then, we compute the likelihood of this event under the impartial anonymous culture hypothesis (defined later). It is known that in three-candidate elections, the Young rule, the Dodgson rule and the Maximin rule always lead to the same top candidate (see Courtin et al., 2012). As one consequence of our analysis, we find that in three-candidate elections, the Young rule, the Dodgson rule and the Maximin rule always lead

\footnotetext{
${ }^{8}$ Also known as the Simpson-Kramer rule(Simpson, 1969; Kramer, 1977) or the Minimax rule (Young, 1977). This rule first determines the support received by each candidate in every pairwise comparisons; the candidate with the greatest minimum support received is the winner.

${ }^{9}$ As proposed by Young (1977), this rule contrasts with the Dodgson rule since rather than to obtain a majority winner by adjacent switches, it proceeds by deletion of voters. The Young rule elects the candidate(s) who needs the fewest number of deletions of voters to become the Condorcet winner.
} 
to the same collective ranking. This last finding highlights a strong connection between these three Condorcet-consistent rules in the sense that there are equivalent in three-candidate elections.

The rest of the paper is structured as follows: Section 2 deals with basic definitions. In Section 3 , we define the stable rules and analyze what we call the divergence on outcomes. Section 4 is devoted to the likelihood of the divergence on outcomes. Section 5 concludes.

\section{Notation and definitions}

\subsection{Binary relation and preferences}

Let $N$ be the set of $n$ voters $(n \geq 2)$ and $A$ the set of $m$ candidates $(m \geq 3)$. We will operate under a framework where voters rank the candidates in a strict way. So, we will write $a P b$ to say that " $a$ is better or strictly preferred to $b$ ". In the sequel, we will simply write, $a b c$ to denote that candidate $a$ is ranked ahead candidate $b$ who is ranked ahead candidate $c$. The preference profile $\pi=\left(P_{1}, P_{2}, \ldots, P_{i}, \ldots, P_{n}\right)$ gives all the linear orders of all the $n$ voters on $A$ where $P_{i}$ is the strict ranking of a given voter $i$. The set of all the preference profiles of size $n$ on $A$ is denoted by $P(A)^{n}$. A voting situation $\tilde{n}=\left(n_{1}, n_{2}, \ldots, n_{t}, \ldots, n_{m !}\right)$ indicates the number of voters for each linear order such that $\sum_{t=1}^{m !} n_{t}=n$. Table 1 describes the voting situation with three candidates.

Table 1: Voting situation on $A=\{a, b, c\}$

\begin{tabular}{llllll}
\hline \hline$n_{1}: a b c$ & $n_{2}: a c b$ & $n_{3}: c a b$ & $n_{4}: c b a$ & $n_{5}: b c a$ & $n_{6}: b a c$ \\
\hline \hline
\end{tabular}

For a given profile $\pi$, when the number of voters who rank $a$ before $b$ (denoted by $n_{a b}$ ) is greater than that of those who rank $b$ before $a$ (denoted by $n_{b a}$ ), $a$ is said to be majority preferred to $b$. We denote this by $a M(\pi) b$ or simply $a M b$ when there is no ambiguity. For $a M b$, we say that candidate $b$ loses the majority pairwise by a margin equal to $n_{a b}-n_{b a}$. If $n_{a b}=n_{b a}$, we say that $a$ and $b$ tie and we denote it by $a T(\pi) b$ or simply $a T b$.

Definition 1. Given $\pi$, candidate $a$ is the Condorcet winner if we have $a M(\pi) b$ for all $b \in A \backslash\{a\}$. Candidate $a$ is the Condorcet loser if we have $b M(\pi) a$ for all $b \in A \backslash\{a\}$.

Definition 2. (Majority cycle). Let $B$ be a nonempty subset of $A$ such that $B=\left\{a_{1}, \ldots, a_{j}, \ldots . a_{l}\right\}$ with $3 \leq l \leq m$. Given the profile $\pi$, the majority relation $M$ is cyclic on $B$ if $a_{j} M a_{j+1} \forall j=$ $1,2, \ldots, l-1$ and $a_{l} M a_{1} ; l$ is the length of the cycle.

Assume that we want to elect a committee of size $g(2 \leq g \leq m-1)$. We denote by $\mathcal{A}^{g}$ the set of all the possible committees of size $g$. A weak Condorcet Committee à la Gerhlein (CCG) is a fixed-size subset of candidates such that no candidate in this subset is defeated in pairwise comparisons by any outside candidate.

Definition 3 (Weak Condorcet Committee à la Gerhlein(CCG)). With $\sharp A=m, C \in \mathcal{A}^{g}$ is a CCG if and only if $\forall x \in C$ we have $y M x$ for no $y \in A \backslash C$.

For a given voting situation, a CCG may not exist given $g$. But there are some cases where one can have more than one CCG (Kamwa, 2015b). 


\section{Stable rules and collective rankings: divergence on out- comes}

Given a profile $\pi$ and $a \in A$, we will denote by $D g(\pi, a), \operatorname{Yg}(\pi, a)$ and $M x n(\pi, a)$, respectively the Dodgson score, the Young-score and the Maximin-score of candidate $a$. All the formal definitions of the stable rules that follows come from Kamwa (2015b). We are only concern in this paper with three of the six stable rules: the DR, the SEO and the YC. For each stable rule, the set of the elected committees is made by the committees with the lowest score of the rule.

If we want to select a committee of $g$ members with the DR rule, the DR-score $D R(\pi, C)$ of a committee $C \in \mathcal{A}^{g}$ is the total number of adjacent switches to make $C$ become a CCG. Given two adjacent candidates $x$ and $y$, an adjacent switch consists in that $x$ and $y$ are permuted ${ }^{10}$ in a given linear order. We denote the DR outcome set by $\mathcal{D} \mathcal{R}_{g}(\pi)$. Let us take a simple example to illustrate this rule.

Example 1. Consider the following voting situation on $A=\{a, b, c\}$ with 15 voters.

$$
7: a c b \quad 3: c b a \quad 5: b a c
$$

The pairwise comparisons and the majority graph of this voting situation are as follows:

Pairwise comparisons
\begin{tabular}{|c|c|c|c|}
\hline \hline$v s$ & $a$ & $b$ & $c$ \\
\hline$a$ & - & 7 & 12 \\
\hline$b$ & 8 & - & 5 \\
\hline$c$ & 3 & 10 & - \\
\hline \hline
\end{tabular}

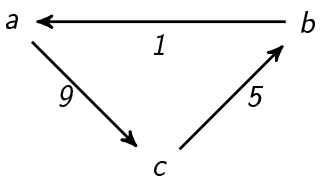

There is no CCG since we have a majority cycle: aMc, $c M b$ and $b M a$. For committee $(a, b)$ to become a $C C G$, it needs to permute $b$ and $c$ in the ranking of three voters among those with acb. So, $D R(\pi,(a, b))=3$. We also get, $D R(\pi,(a, c))=1$ and $D R(\pi,(b, c))=5$. Thus, $\mathcal{D R}_{g}(\pi)=\{(a, c)\}$.

If we compte the Dodgson-scores for each candidate, we will get $D g(\pi, a)=1, D g(\pi, b)=3$ and $D g(\pi, c)=5$. So, the collective ranking of the Dodgson rule is abc since candidates are ranked from the one with the lowest score to the one with the greatest score. So, the Dodgson rule will recommend $(a, b)$ as the two-member committee elected while the DR recommends $(a, c)$.

For a given profile $\pi$, the SEO-score $(S E O(\pi, C))$ of committee $C \in \mathcal{A}^{g}$ is the maximum number of voters by which a candidate not in $C$ is majority preferred to a candidate in $C$. We denote the SEO-outcome set by $\mathcal{S E O}_{g}(\pi)$. If we apply the SEO rule to the voting situation of Example 1, we have $\operatorname{SEO}(\pi,(a, b))=10$ since $n_{c b}=10$; we also have $\operatorname{SEO}(\pi,(a, c))=8$ and $\operatorname{SEO}(\pi,(b, c))=12$. Thus, $\mathcal{S E O}_{g}(\pi)=\{(a, c)\}$. If we compute the Maximin scores, we get $\operatorname{Mxn}(\pi, a)=7, \operatorname{Mxn}(\pi, b)=5$ and $\operatorname{Mxn}(\pi, c)=3$. Then, $a b c$ is the Maximin ranking; so, the Maximin rule will recommend $(a, b)$ as the two-member committee elected while the SEO recommends $(a, c)$.

In contrast with the $\mathrm{DR}$ rule, the $\mathrm{YC}$ rule selects the committee(s) that need the fewest deletions of voters to become a CCG. For a given committee, its YC-score is simply the number of deletions needed to become the CCG. We denote by $\mathcal{Y} \mathcal{C}_{g}(\pi)$ the YC-outcome set. With the voting situation of Example 1, we have $Y C(\pi,(a, c))=1$ since it only needs the deletion of one voter that ranks $b$ before $a$. We also get $Y C(\pi,(a, b))=5$ and $Y C(\pi,(b, c))=9$. Thus, $\mathcal{Y C}_{g}(\pi)=\{(a, c)\}$. If we

\footnotetext{
${ }^{10}$ With simple words, in a linear order, $x$ takes the place of $y$ and $y$ takes that of $x$.
} 
compute the Young scores, we get $\operatorname{Yg}(\pi, a)=2, \operatorname{Yg}(\pi, b)=6$ and $\operatorname{Yg}(\pi, c)=10$. Then, $a b c$ is the Young ranking and the Young rule will recommend $(a, b)$ as the two-member committee elected while the $\mathrm{YC}$ rule recommends $(a, c)$.

It comes from Example 1 that given a voting situation, a stable rule can select a committee totally different from the one recommended by the corresponding classical rule. Thereby, this defines what we call the divergence on outcomes. It could be interesting to know if the divergence on outcomes is just a rare oddity or betray a more generalized behavior. This is the purpose of the next section. First of all, we have to pinpoint the conditions that guarantee that this event can occur or not. Before that let us state some intermediate results.

Courtin et al. (2012) have shown that the Dodgson rule, the Maximin rule and the Young rule always select the same winner in three-candidate elections. Proposition 1 strengthens this result as a consequence of our analysis.

Proposition 1. In three-candidate elections, the Dodgson rule, the Maximin rule and the Young rule always lead to the same collective ranking. As a consequence, if there is a Condorcet loser, he is never ranked first by any of these rules.

Proof. See appendix.

It is well established in the literature ${ }^{11}$ that in one-winner election, the Dodgson rule, the Maximin rule and the Young rule can select the Condorcet loser when there is no Condorcet winner. According to Proposition 1, this well-known is valid with at least 4 candidates. So, with more than three candidates, the second part of Proposition 1 would not hold. Let us use an example to show that the first part of Proposition 1 would not hold in four-candidates elections.

Example 2. Assume $A=\{a, b, c, d\}$ and 9 voters.

\begin{tabular}{c} 
preferences \\
\hline \hline $2: a b c d$ \\
$2: a d c b$ \\
$2: c b d a$ \\
$3: d b c a$ \\
\hline \hline
\end{tabular}

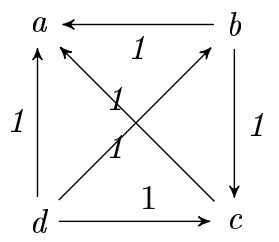

Pairwise comparisons
\begin{tabular}{|c|c|c|c|c|}
\hline \hline$v s$ & $a$ & $b$ & $c$ & $d$ \\
\hline$a$ & - & 4 & 4 & 4 \\
\hline$b$ & 5 & - & 5 & 4 \\
\hline$c$ & 5 & 4 & - & 4 \\
\hline$d$ & 5 & 5 & 5 & - \\
\hline \hline
\end{tabular}

Here, $d$ is the Condorcet winner. If we compute the scores, with the Dodgson rule we get $D g(\pi, a)=$ $3, D g(\pi, b)=1, D g(\pi, c)=2$ and $D g(\pi, d)=0$; with the Young rule we get $Y g(\pi, a)=Y g(\pi, b)=$ $2, Y g(\pi, c)=6$ and $Y g(\pi, d)=0$; with the Maximin rule we get $\operatorname{Mxn}(\pi, a)=\operatorname{Mxn}(\pi, b)=$ $\operatorname{Mxn}(\pi, c)=4$ and $\operatorname{Mxn}(\pi, d)=5$. So, dbac is the Dodgson ranking, $d(a, b) c$ is the Young ranking ${ }^{12}$ and $d(a, b, c)$ is the Maximin ranking. Thus, with more than three candidates, the Maximin rule, the Young rule and the Dodgson rule may not lead to the same ranking.

We know from Kamwa (2015b, Theorem 1) that in three-candidate elections, the DR rule, the $\mathrm{SEO}$ rule and the YC rule are equivalent since they always select the same committee. So, from this point, we will denote by " $\mathcal{S}$ " one these rules and by $\mathcal{V}$ the corresponding classical rule since Proposition 1 tells us that the Dodgson rule, the Maximin rule and the Young rule are equivalent in three-candidate elections.

\footnotetext{
${ }^{11}$ See Fishburn (1977); Kamwa (2015a).

${ }^{12} \mathrm{By} d(a, b) c$, we mean that $d$ is at the top and is followed by $a$ and $b$ who tie since they get the same score.
} 
Proposition 2. Consider a preference profile with three candidates. The stable rule $\mathcal{S}$ never elects a two-member committee made of the bottom candidates of the $\mathcal{V}$ ranking.

Proof. See appendix.

With the following example, we show that Proposition 2 would not hold in four-candidates elections and three-member committee $(g=3)$.

Example 3. Assume $A=\{a, b, c, d\}$ and 33 voters.

\begin{tabular}{cc}
\multicolumn{2}{c}{ preferences } \\
\hline \hline $6: a b c d$ & $10: c d b a$ \\
$5: b c d a$ & $1: b a c d$ \\
$10: a d b c$ & $1: d c a b$ \\
\hline \hline
\end{tabular}

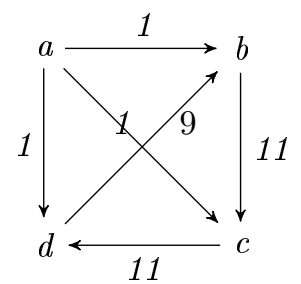

Pairwise comparisons
\begin{tabular}{|c|c|c|c|c|}
\hline \hline$v s$ & $a$ & $b$ & $c$ & $d$ \\
\hline$a$ & - & 17 & 17 & 17 \\
\hline$b$ & 16 & - & 22 & 12 \\
\hline$c$ & 16 & 11 & - & 22 \\
\hline$d$ & 16 & 21 & 11 & - \\
\hline \hline
\end{tabular}

With this profile, a is the Condorcet winner. The reader can easily check that the Maximin rule, the Dodgson rule and the Young rule lead to the same collective ranking: abcd or abdc as $c$ and $d$ get the same score under each of the rules. When computing the scores for our stable rules for three-member committee $(g=3)$, we get what follows:

\begin{tabular}{|c|c|c|c|}
\cline { 2 - 4 } \multicolumn{1}{c|}{} & \multicolumn{3}{c|}{ Scores for $g=3$} \\
\hline commitees & DR & SEO & $Y C$ \\
\hline$\{a, b, c\}$ & 5 & 21 & 9 \\
\hline$\{a, b, d\}$ & 6 & 22 & 11 \\
\hline$\{a, c, d\}$ & 6 & 22 & 11 \\
\hline$\{b, c, d\}$ & 3 & 17 & 1 \\
\hline
\end{tabular}

The DR, the YC and the SEO rules all select the same committee: $(b, c, d)$. Candidate a, the Condorcet winner is not appointed ${ }^{13}$. The elected committee is then made of the bottom candidates of the Maximin/Dodgson/Young ranking.

For three-candidate elections, Theorem 1 characterizes all the voting situations in which a twomember committee elected with a stable rule $\mathcal{S}$ is not always made of the top two ranked candidates of the rule $\mathcal{V}$.

Theorem 1. Consider a voting profile with three candidates. The divergence on outcomes between $\mathcal{S}$ and $\mathcal{V}$ for two-member committee elections may occur if and only if (i)- there is a Condorcet winner and a Condorcet loser simultaneously, or (ii)- there is a majority cycle among candidates.

Proof. See appendix.

The following example shows that Theorem 1 would not hold in four-candidate elections and three-member committee.

Example 4. Assume $A=\{a, b, c, d\}$ and let us consider the following profiles:

\footnotetext{
${ }^{13}$ Kamwa (2015b) showed that the DR, the YC and the SEO rules fail the Condorcet winner criterion.
} 


\begin{tabular}{ccc}
\multicolumn{3}{c}{ profile 1 } \\
\hline \hline $1: a d b c$ & $1:$ bacd & $1: c a b d$ \\
\hline \hline
\end{tabular}

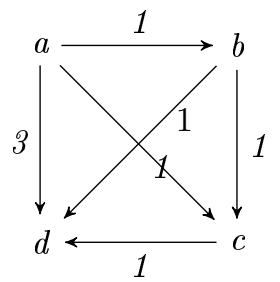

profile 2

\begin{tabular}{llll}
\hline \hline $2: a d b c$ & $1: b a c d$ & $2: b c a d$ & $2: c a d b$ \\
\hline \hline
\end{tabular}

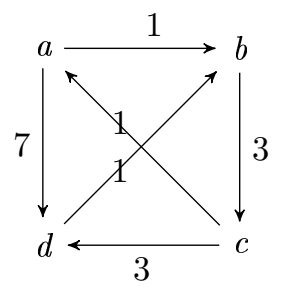

With the profile 1, a is the Condorcet winner and $d$ the Condorcet loser. So, committee $(a, b, c)$ is the $C C G$ for $g=3$ and it is the unique committee selected by the $Y C$, the DR and the SEO rules. The reader can easily check that with this profile, the Dodgson ranking, the Young ranking and the Maximin ranking is the same with candidate $d$ at the bottom and the others at the top since they tie. In this case, there is no divergence. This is also the case with the profile 2: there is a majority cycle among the candidates and committee $(a, b, c)$ unique elected committee of the $Y C$, the DR and the SEO rules. The Dodgson rule, the Young rule and the Maximin rule lead to the same ranking such that $d$ at the bottom and $a$ and $b$ are at the top since they tie.

We can now use Theorem 1 to evaluate the likelihood of the divergence. This is what we do in the next section.

\section{Evaluating the divergence on outcomes}

\subsection{Necessary and sufficient conditions}

Using the labels of Table 1, Lemmata 1 and 2 that follow explicit what we have stated in Theorem 1.

Lemma 1. Consider the labels of Table 1. Assume that candidate a is the Condorcet winner while candidate $c$ is the Condorcet loser. The rules $\mathcal{S}$ and $\mathcal{V}$ may diverge on outcomes if

$$
\left\{\begin{array} { l } 
{ n _ { b a } < n _ { a b } } \\
{ n _ { c a } < n _ { a c } } \\
{ n _ { c b } < n _ { b c } } \\
{ n _ { b a } \leq n _ { c a } } \\
{ n _ { c a } \leq n _ { c b } }
\end{array} \quad \Leftrightarrow \quad \left\{\begin{array}{l}
-n_{1}-n_{2}-n_{3}+n_{4}+n_{5}+n_{6}<0 \\
-n_{1}-n_{2}+n_{3}+n_{4}+n_{5}-n_{6}<0 \\
-n_{1}+n_{2}+n_{3}+n_{4}-n_{5}-n_{6}<0 \\
-n_{3}+n_{6} \leq 0 \\
-n_{2}+n_{5} \leq 0 \\
n_{1}+n_{2}+n_{3}+n_{4}+n_{5}+n_{6}=n
\end{array}\right.\right.
$$


or if

$$
\left\{\begin{array} { l } 
{ n _ { b a } < n _ { a b } } \\
{ n _ { c a } < n _ { a c } } \\
{ n _ { c b } < n _ { b c } } \\
{ n _ { b a } \leq n _ { c b } } \\
{ n _ { c b } \leq n _ { c a } }
\end{array} \quad \Leftrightarrow \quad \left\{\begin{array}{l}
-n_{1}-n_{2}-n_{3}+n_{4}+n_{5}+n_{6}<0 \\
-n_{1}-n_{2}+n_{3}+n_{4}+n_{5}-n_{6}<0 \\
-n_{1}+n_{2}+n_{3}+n_{4}-n_{5}-n_{6}<0 \\
-n_{2}-n_{3}+n_{5}+n_{6} \leq 0 \\
n_{2}-n_{5} \leq 0 \\
n_{1}+n_{2}+n_{3}+n_{4}+n_{5}+n_{6}=n
\end{array}\right.\right.
$$

Proof. In Equations 1 and 2, the reader can check that the first three inequalities guarantee that candidate $a$ is the Condorcet winner and that candidate $c$ is the Condorcet loser; thus, $\mathcal{S}$ will select $(a, b)$. Since we know by Proposition 2 that the Condorcet loser $c$ cannot be ranked first by $\mathcal{V}$, it follows that $a c b$ is the only ranking we can have such that the two-member committee elected by $\mathcal{S}$ is not made of the top two ranked candidates of $\mathcal{V}$. The worst support of candidate $b$ is equal to $n_{b a}$ in front of candidate $a$; for candidate $c$, it could be $n_{c a}$ or $n_{c b}$. So, if $c$ is ranked before $b$ we will have $n_{c a} \geq n_{b a}$ with $n_{c a} \leq n_{c b}$ or $n_{c b} \geq n_{b a}$ with $n_{c b} \leq n_{c a}$. This completes the proof.

Lemma 2. Consider the labels of Table 1 and assume that there is a majority cycle such that aMb, $b M c$ and $c M a$. The rules $\mathcal{S}$ and $\mathcal{V}$ may diverge on outcomes if

$$
\left\{\begin{array} { l } 
{ n _ { b a } < n _ { a b } } \\
{ n _ { c b } < n _ { b c } } \\
{ n _ { a c } < n _ { c a } } \\
{ n _ { c a } \leq n _ { b c } } \\
{ n _ { c a } \leq n _ { a b } } \\
{ n _ { b a } \leq n _ { c b } }
\end{array} \Leftrightarrow \quad \left\{\begin{array}{l}
-n_{1}-n_{2}-n_{3}+n_{4}+n_{5}+n_{6}<0 \\
-n_{1}+n_{2}+n_{3}+n_{4}-n_{5}-n_{6}<0 \\
n_{1}+n_{2}-n_{3}-n_{4}-n_{5}+n_{6}<0 \\
-n_{1}+n_{3}+n_{4}-n_{6} \leq 0 \\
-n_{1}-n_{2}+n_{4}+n_{5} \leq 0 \\
-n_{2}-n_{3}+n_{5}+n_{6} \leq 0 \\
n_{1}+n_{2}+n_{3}+n_{4}+n_{5}+n_{6}=n
\end{array}\right.\right.
$$

Proof. Using the development of the previous section, the reader can easily check that the three first inequalities defines the assumed cycle among candidates. Let us assume that $(a, b)$ is selected by $\mathcal{S}$. So, we have $n_{c a} \leq n_{b c}$ (i) and $n_{c a} \leq n_{a b}$ (ii). The worst support of candidate $a, b$ and $c$ are respectively equal to $n_{a c}, n_{b a}$ and $n_{c b}$. The rules $\mathcal{S}$ and $\mathcal{V}$ may diverge on outcomes only if the worst support of candidate $a$ is less to that of candidates $b$ and $c$ or if the worst support of candidate $b$ is less than that of candidates $a$ and $c$. This respectively leads to $n_{a c} \leq n_{b a}$ (iii) and $n_{a c} \leq n_{c b}$ (iv) or $n_{b a} \leq n_{a c}$ (v) and $n_{b a} \leq n_{c b}$ (vi).

Suppose that the committee of two-member selected by $\mathcal{S}$ is not made of the top two candidate of the ranking of $\mathcal{V}$; this means that we have ((i) and (ii) and (iii) and (iv)) or ((i) and (ii) and (v) and (vi)). Nevertheless, (ii) and (iii) can not hold simultaneously; thus, we get the supposed disagreement only with ((i) and (ii) and (v) and (vi)). Since (ii) is equivalent to (v), the relations that define the divergence are ((i) and (ii) and (vi)); added to the first three inequalities of equation 3 , this completes the proof.

We are now able to undertake the computation of the likelihood of the divergence between a committee elected with a stable rule $\mathcal{S}$ and the one recommended by the ranking of the rule $\mathcal{V}$ from which the stable rule $\mathcal{S}$ is derived. For this, we use the Impartial Anonymous Culture (IAC). 


\subsection{The IAC model}

The impartial anonymous culture assumption (IAC) is one of the most used assumptions in the social choice literature when computing the likelihood of voting events ${ }^{14}$. This assumption was first proposed by Gehrlein and Fishburn (1976). Under IAC, each voting situation is equally likely. The likelihood of a given event $X$ is calculated in respect with the following ratio:

$$
\operatorname{Pr}(X)=\frac{\text { Number of voting situations in which event } X \text { is likely }}{\text { Total number of possible voting situations }}
$$

It is known that the total number of possible voting situations in three-candidate elections is given by the following five-degree polynomial in $n$,

$$
\Psi(n)=C_{n+3 !-1}^{n}=\frac{(n+5) !}{n ! 5 !}
$$

To compute the number of voting situations in which event $X$ is likely to occur, many techniques and algorithms have been proposed to carry out such computations. Without been exhaustive, we can list among others, Barvinok (1994); Clauss and Loechner (1998); Barvinok and Pommersheim (1999); Gehrlein and Fishburn (1976); Haung and Chua (2000); Ehrhart (1962, 1967); Verdoolaege et al. (2004)). Among the proposed algorithms, we use the parameterized Barvinok's algorithm developed by Verdoolaege et al. (2004) to compute $\Phi(n)$ the number of voting situations in which the divergence is likely to occur. This algorithm proceeds by the enumeration of the Ehrhart polynomials (Ehrhart $(1962,1967))$. We are not going to recall the Ehrhart theory and all the attached algorithms. The reader can refer to Ehrhart (1962, 1967); Barvinok (1994); Barvinok and Pommersheim (1999); Bruynooghe et al. (2005); Clauss and Loechner (1998); Lepelley et al. (2008); Verdoolaege et al. (2004). We say some words on the Verdoolaege et al. (2004)'s methodology in the appendix.

\subsection{Probabilities of divergence}

For each of the Equations 1,2 and 3, the computer program returns the results in five-degree Ehrhart's polynomials ${ }^{15}$.

- With Equation 1, we get the following polynomial for $n \geq 1$ :

$$
\Phi_{S_{1}}(n)=\frac{1}{3840} n^{5}+\left[\frac{1}{192}, \frac{5}{768}\right]_{n} n^{4}+\left[\frac{7}{192}, \frac{23}{384}\right]_{n} n^{3}+\left[\frac{5}{48}, \frac{95}{384}\right]_{n} n^{2}+\left[\frac{1}{10}, \frac{563}{1280}\right]_{n} n+\left[0, \frac{63}{256}\right]_{n}
$$

- With Equation 2, we get the following polynomial for $n \geq 1$ :

$$
\begin{gathered}
\Phi_{S_{2}}(n)=\frac{1}{7680} n^{5}+\left[\frac{5}{1536}, \frac{1}{256}\right]_{n} n^{4}+\left[\frac{11}{384}, \frac{11}{256}\right]_{n} n^{3}+\left[\frac{5}{48}, \frac{27}{128}\right]_{n} n^{2} \\
+\left[\frac{2}{15}, \frac{3389}{7680}\right]_{n} n+\left[0, \frac{77}{256}, \frac{1}{32}, \frac{69}{256}\right]_{n}
\end{gathered}
$$

\footnotetext{
${ }^{14}$ For more on the other assumptions, the reader may see Gehrlein and Lepelley (2010).

${ }^{15}$ For $\Phi(n)=\left[-\frac{1}{12}, \frac{1}{18}, \frac{1}{6},-\frac{7}{36}\right]_{n} n$, this means that $\Phi(n)$ is a periodic number with 4 as the period; so, $\Phi(n)=$ $-\frac{1}{12} n$ for $n \equiv 0 \bmod 4, \Phi(n)=\frac{1}{18} n$ for $n \equiv 1 \bmod 4, \Phi(n)=\frac{1}{6} n$ for $n \equiv 2 \bmod 4$ and $\Phi(n)=-\frac{7}{36} n$ for $n \equiv 3 \bmod 4$.
} 
- With Equation 3, we get the following polynomial for $n \geq 3$ :

$$
\begin{gathered}
\Phi_{S_{3}}(n)=\frac{1}{23040} n^{5}+\left[\frac{1}{1536}, \frac{1}{768}\right]_{n} n^{4}+\left[\frac{5}{3456}, \frac{91}{6912}\right]_{n} n^{3}+\left[-\frac{1}{96}, \frac{19}{384}\right]_{n} n^{2}+\left[-\frac{1}{40}, \frac{101}{2560}\right]_{n} n \\
+\left[0,-\frac{715}{6912}, \frac{59}{864},-\frac{9}{256},-\frac{1}{27},-\frac{203}{6912}, \frac{1}{32},-\frac{499}{6912}, \frac{1}{27},-\frac{17}{256},-\frac{5}{864}, \frac{13}{6912}\right]_{n}
\end{gathered}
$$

If we denote by $\operatorname{Pr}(\operatorname{Div}, n)$ the probability of the divergence on outcomes, it is equal to ${ }^{16}$

$$
\operatorname{Pr}(\operatorname{Div}, n)=\frac{3 \Phi(n)}{\Psi(n)}
$$

We have shown that the divergence on outcomes is likely to occur only if there is a Condorcet winner and a Condorcet loser simultaneously or if there is majority cycle. Thus, the probability $\operatorname{Pr}(\operatorname{Div}, n)$ is equal to

$$
\operatorname{Pr}(\operatorname{Div}, n)=\operatorname{Pr}\left(\operatorname{Div}_{1}, n\right)+\operatorname{Pr}\left(\operatorname{Div}_{1}, n\right)
$$

with $\operatorname{Pr}\left(\operatorname{Div}_{1}\right)$ the likelihood of the divergence when there is a Condorcet winner and a Condorcet loser simultaneously and $\operatorname{Pr}\left(\operatorname{Div}_{2}, n\right)$ the likelihood of the divergence when there is a majority cycle.

$$
\begin{gathered}
\operatorname{Pr}\left(\operatorname{Div}_{1}, n\right)=3\left(\frac{\Phi_{S_{1}}(n)+\Phi_{S_{2}}(n)}{\Psi(n)}\right) \\
\operatorname{Pr}\left(\operatorname{Div}_{2}, n\right)=3\left(\frac{\Phi_{S_{3}}(n)}{\Psi(n)}\right)
\end{gathered}
$$

Also, we denote by $\widehat{\operatorname{Pr}}\left(\operatorname{Div}_{1}, n\right), \widehat{\operatorname{Pr}}\left(\operatorname{Div}_{2}, n\right)$ and $\operatorname{Pr}(\operatorname{Div}, n)$ the corresponding conditional probabilities such that

$$
\begin{aligned}
& \widehat{\operatorname{Pr}}\left(\operatorname{Div}_{1}, n\right)=\frac{\operatorname{Pr}\left(\operatorname{Div}_{1}, n\right)}{1-\operatorname{Pr}_{c y}(n)} \\
& \widehat{\operatorname{Pr}}\left(\operatorname{Div}_{2}, n\right)=\frac{\operatorname{Pr}\left(\operatorname{Div}_{2}, n\right)}{\operatorname{Pr}_{c y}(n)}
\end{aligned}
$$

where $\operatorname{Pr}_{c y}(n)$ is the probability that there is a majority cycle with three candidates and $n$ voters. The values of $\operatorname{Pr}_{c y}(n)$ can be drawn from Gehrlein and Lepelley (2010, p.21). To be more precise, $\widehat{\operatorname{Pr}}\left(\operatorname{Div}_{1}, n\right)$ is the conditional probability of the divergence on outcomes given that there is a Condorcet winner and a Condorcet loser simultaneously and $\widehat{\operatorname{Pr}}\left(\operatorname{Div}_{2}, n\right)$ is the conditional probability of the divergence on outcomes given that there is a majority cycle. We report in Table 2 the corresponding probabilities of the divergence on outcomes given the size of the electorate.

What comes from Table 2 is that with 8 voters, if there is a Condorcet winner and a Condorcet loser simultaneously, the two-member committee elected with the $\mathrm{SEO} / \mathrm{DR} / \mathrm{YC}$ rule and that recommended by the Maximin/Dodgson/Young rule diverge in $22.38 \%$ of cases; they diverge in $0.93 \%$ of cases if there is a majority cycle among candidates. Then, with 8 voters, the overall probability

\footnotetext{
${ }^{16}$ In order to obtain the conditions of the Lemmata 1 and 2, we have assumed a particular situation involving a pair of candidates, assume $\{a, b\}$ for example. To get the total probability, we also need to consider the other pairs: $\{a, c\}$ and $\{b, c\}$. It is worth noting that cases are symmetrical; it is therefore sufficient to reason on only one case and multiply the result by 3 to obtain the desired probability.
} 
Table 2: (Conditional) Probabilities of the divergence on outcomes

\begin{tabular}{||c|c|c||c||c||c||c||}
\hline \hline$n$ & $\operatorname{Pr}\left(\operatorname{Div}_{1}, n\right)$ & $\operatorname{Pr}\left(\operatorname{Div}_{2}, n\right)$ & $\operatorname{Pr}(\operatorname{Div}, n)$ & $\operatorname{Pr}_{c y}(n)$ & $\widehat{\operatorname{Pr}}\left(\operatorname{Div}_{1}, n\right)$ & $\widehat{\operatorname{Pr}}\left(\operatorname{Div}_{2}, n\right)$ \\
\hline \hline 8 & 0.2238 & 0.0093 & 0.2331 & 0.3007 & 0.3200 & 0.0310 \\
9 & 0.3177 & 0.0375 & 0.3552 & 0.0530 & 0.3354 & 0.7068 \\
10 & 0.2118 & 0.0110 & 0.2228 & 0.2657 & 0.2884 & 0.0410 \\
11 & 0.2871 & 0.0343 & 0.3214 & 0.0577 & 0.3047 & 0.5952 \\
12 & 0.2027 & 0.0121 & 0.2148 & 0.2398 & 0.2666 & 0.0505 \\
13 & 0.2654 & 0.0319 & 0.2973 & 0.0588 & 0.2820 & 0.5419 \\
14 & 0.1956 & 0.0129 & 0.2085 & 0.2198 & 0.2507 & 0.0587 \\
15 & 0.2492 & 0.0300 & 0.2792 & 0.0596 & 0.2650 & 0.5032 \\
16 & 0.1899 & 0.0134 & 0.2033 & 0.2039 & 0.2385 & 0.0658 \\
17 & 0.2367 & 0.0285 & 0.2652 & 0.0602 & 0.2519 & 0.4731 \\
18 & 0.1853 & 0.0138 & 0.1991 & 0.1909 & 0.2290 & 0.0724 \\
19 & 0.2268 & 0.0272 & 0.2540 & 0.0606 & 0.2414 & 0.4496 \\
20 & 0.1814 & 0.0141 & 0.1955 & 0.1801 & 0.2213 & 0.0784 \\
$\vdots$ & $\vdots$ & $\vdots$ & $\vdots$ & $\vdots$ & $\vdots$ & $\vdots$ \\
31 & 0.1937 & 0.0230 & 0.2167 & 0.0617 & 0.2064 & 0.3722 \\
$\vdots$ & $\vdots$ & $\vdots$ & $\vdots$ & $\vdots$ & $\vdots$ & $\vdots$ \\
50 & 0.1584 & 0.0153 & 0.1737 & 0.1146 & 0.1789 & 0.1338 \\
$\vdots$ & $\vdots$ & $\vdots$ & $\vdots$ & $\vdots$ & $\vdots$ & $\vdots$ \\
75 & 0.1626 & 0.0187 & 0.1813 & 0.0623 & 0.1734 & 0.3005 \\
$\vdots$ & $\vdots$ & $\vdots$ & $\vdots$ & $\vdots$ & $\vdots$ & $\vdots$ \\
101 & 0.1569 & 0.0179 & 0.1748 & 0.0624 & 0.1673 & 0.2874 \\
$\vdots$ & $\vdots$ & $\vdots$ & $\vdots$ & $\vdots$ & $\vdots$ & $\vdots$ \\
$\vdots$ & $\vdots$ & $\vdots$ & $\vdots$ & $\vdots$ & $\vdots$ & $\vdots$ \\
1001 & 0.1423 & 0.0159 & 0.1581 & 0.0625 & 0.1517 & 0.2537 \\
$\vdots$ & $\vdots$ & $\vdots$ & $\vdots$ & $\vdots$ & $\vdots$ & $\vdots$ \\
$\vdots$ & $\vdots$ & $\vdots$ & $\vdots$ & $\vdots$ & $\vdots$ & $\vdots$ \\
$\infty$ & 0.1406 & 0.0156 & 0.1563 & 0.0667 & 0.1506 & 0.2339 \\
\hline \hline & & & & & & \\
\hline
\end{tabular}


of divergence is equal to $23.31 \%$. As the electorate tends to the infinity, the limit probability of divergence is equal to $15.63 \%$. We notice that with more than 8 voters, the likelihood of the divergence when there simultaneously exists a Condorcet winner and a Condorcet loser tends to be nine times greater than that of the divergence when there is a majority cycle. Overall, the probability of divergence tends to decrease with the size of the electorate.

According to what we get in the proof of Theorem 1, $\operatorname{Pr}\left(\operatorname{Div}_{1}\right)$ is also the probability in threecandidate elections that each of the Maximin rule, the Dodgson rule and the Young rule fails to elect the CCG when it exists. So, when the electorate is very large, the Condorcet/Dodgson/Young rule fails to elect the Condorcet committee à la Gehrlein when it exists in $14.06 \%$ of cases.

\section{Concluding remarks}

In this paper, we focused on the relationships that exists in three-candidate elections between three stable rules for committee elections (the DR rule, the SEO rule and the YC rule) and the classical rules from which each of these stable rules are derived. The DR rule is adapted from the Dodgson rule, the SEO rule from the Maximin rule and the YC rule from the Young rule. The departure point of our analysis was the fact that the DR rule, the SEO rule and the $\mathrm{YC}$ rule are equivalent in three-candidate elections as they also select the same two-member committee (Kamwa, 2015b). There are some cases where a committee selected by a stable rule may differ from the committee made of the best candidates of the corresponding classical rule from which the stable rule is adapted. We called this the divergence on outcomes.

Courtin et al. (2012) showed in three-candidate elections that the Dodgson rule, the Maximin rule and the Young rule always select the same winner. We supplement this result by showing that these three Condorcet consistent rules always lead to the same collective ranking. This helped us to characterize all the voting situations under which the divergence on outcomes is likely to occur: if there is a Condorcet winner and a Condorcet loser simultaneously or if there is a majority cycle. We used this characterization in order to evaluate the likelihood of this voting event. It came that

the probability of this event is not negligible at all. From the computation of the likelihood of the divergence when there is simultaneously a Condorcet winner and a Condorcet loser, we derive how often the three Condorcet-consistent rules we focused on (the Dodgson rule, the Maximin rule and the Young rule) fail to select the Condorcet Committee à la Gehrlein. We noticed that the likelihood of the divergence when there simultaneously exists a Condorcet winner and a Condorcet loser tends to be nine times greater than that of the divergence when there is a majority cycle.

However, our conclusions can not be generalized since the analysis was restricted to threecandidate cases. Computations with more than three candidates would have tell us more on how our results can be affected. But, this can become an untractable and cumbersome task since computing the scores of the the Dodgson rule (the Maximin rule, the Young rule) is intractable (Bartholdi et al., 1989; Betzler et al., 2008; Caragiannis et al., 1998; Hemaspaandra et al., 1997). Monte-Carlo simulations can be seen as a way to circumvent this difficulty. 


\section{Appendix}

\section{A-Proof of Proposition 1}

We admit that there can be another way to do the proof but we use a simple approach to be suited to the proof of Theorem 1. Our methodology goes from the possible outcomes of the pairwise comparisons between candidates in $A=\{a, b, c\}$. There are six such conceivable configurations or cases:

1. There is a Condorcet winner while the other candidates tie.

2. There is a Condorcet loser while the other candidates tie.

3. There is both a Condorcet winner and a Condorcet loser.

4. Two candidates tie and one of them majority dominates the third candidate.

5. There is majority cycle.

6. All the candidate tie.

Obviously, the last case is irrelevant. Let us examine each of the other cases.

Case 1 (There is a Condorcet winner while the other candidates tie). Let us take $a M(\pi) b, a M(\pi) c$ and $b T(\pi) c$. Here, candidate $a$ is the Condorcet winner and $n$ is even. Assume that the Dodgson ranking is $a b c$ while that of the Maximin is $a c b$. With $a b c$ as the Dodgson ranking, this implies $n_{b a}>n_{c a}$ (i); for $a c b$ as the Maximin ranking, we get $n_{b a}<n_{c a}$ (ii) which contradicts (i). Thus, the Maximin ranking and the Dodgson ranking are the same in this case. Notice that since $b$ et $c$ tie and that $n$ is even, we have $\operatorname{Yg}(\pi, b)=n-2 n_{b a}+1$ and $\operatorname{Yg}(\pi, c)=n-2 n_{c a}+1$; then, $\operatorname{Yg}(\pi, b)-\operatorname{Yg}(\pi, c)=2\left(n_{c a}-n_{b a}\right)$. If we assume that $a c b$ is the Young ranking, this means that $\operatorname{Yg}(\pi, b)-\operatorname{Yg}(\pi, c)>0$ equivalent to $n_{c a}>n_{b a}$ which contradicts (i). The Maximin ranking and the Dodgson ranking and the Young ranking are the same in this case.

Case 2 (There is a Condorcet loser while the other candidates tie). $a T(\pi) b, a M(\pi) c$ and $b M(\pi) c$. Candidate $c$ is the Condorcet loser. It follows that $a$ and $b$ will have the same Maximin score (equal to $\frac{n}{2}$ ) smaller than that of $c$ which is less than $\frac{n}{2}$. Also, candidates $a$ and $b$ have the same Dodgson score (equal to 1 here) which is smaller than that of candidate $c$. The same holds with the Young rule since each of candidates $a$ and $b$ need only one deletion to become a Condorcet winner while $c$ will need more than that. Then, the Maximin ranking and the Dodgson ranking and the Young ranking are the same in this case.

Case 3 (There is both a Condorcet winner and a Condorcet loser). Assume $a M(\pi) b, a M(\pi) c$ and $b M(\pi) c$. Candidate $a$ is the Condorcet winner and candidate $c$ is the Condorcet loser. The Dodgson, the Young and the Maximin scores of candidate $c$ are respectively lower than those of candidate $b$. Then, the Maximin ranking and the Dodgson ranking are the same in this case. It comes from Cases 2 and 3 that in three-candidate elections, when there is a Condorcet loser, he is never top ranked neither by the Dodgson rule nor by the Maximin rule and nor by the Young rule.

Case 4 ( Two candidates tie and one of them majority dominates the third candidate). Assume $a T(\pi) b, a M(\pi) c$ and $b T(\pi) c$. This case is straightforward since candidates $a$ and $b$ will score the same and better than $c$ under each rule. Thus, the ranking will be the same under each rule. 
Case 5 (There is majority cycle). Let us assume $a M(\pi) b, b M(\pi) c, c M(\pi) a$ and that the Dodgson ranking is $a b c$; this implies that $n_{a c}>n_{b a}>n_{c b}$ (i). Suppose that the Maximin ranking is $a c b$. This is equivalent to $n_{a c}>n_{c b}>n_{b a}$ : this contradicts (i). Thus, the Maximin ranking and the Dodgson ranking are the same in this case. Also, if we assume that the Young ranking is $a c b$, it means that $\operatorname{Yg}(\pi, b)-\mathrm{Yg}(\pi, c)>0$ equivalent to $n_{c b}>n_{b a}$ which contradicts (i). Thus, in this case the ranking is the same for all the rules.

\section{B-Proof of Proposition 2}

For the proof, we reconsider all the possible cases we got in the proof of Proposition 1. In each of these cases, we have assumed that $a b c$ is the collective ranking of $\mathcal{V}$. Since there is at least one CCG in the Cases 1 to 4 , it is obvious that $(b, c)$ is never selected by $\mathcal{S}$. We only have to investigate Case 5 with the majority cycle where $a M(\pi) b, b M(\pi) c$ and $c M(\pi) a$. We know that with $a b c$ as the collective ranking of $\mathcal{V}$, we get $n_{a c}>n_{b a}>n_{c b}$. Assume that $(b, c)$ is selected by $\mathcal{S}$. This means that $\mathcal{S}(\pi,(a, b))-\mathcal{S}(\pi,(b, c))>0$. Since $\mathcal{S}(\pi,(a, b))-\mathcal{S}(\pi,(b, c))=n_{b a}-n_{a c}>0$ : this contradicts (i) an it follows that $(b, c)$ is not selected by $\mathcal{S}$.

\section{C-Proof of Theorem 1}

Let us reconsider each of the possible cases we got in the proof of Proposition 1. Case 6 is still irrelevant. Since there are CCG, committees $(a, b)$ and $(a, c)$ are selected by $\mathcal{S}$ in Cases 1 and 4 and committee $(a, b)$ is selected in Case 2. By Proposition 1 we know that $a b c$ or $a c b$ are the possible rankings of $\mathcal{V}$ in Case 1; $a b c$ or $b a c$ in Cases 2 and 4. So, no matter what the ranking of $\mathcal{V}$ is, $\mathcal{S}$ and $\mathcal{V}$ do not diverge.

In Example 1, we have a majority cycle and committee $(a, c)$ is selected by $\mathcal{S}$ while the collective ranking of $\mathcal{V}$ is $a b c$. So, in presence of a majority cycle, $\mathcal{S}$ and $\mathcal{V}$ may diverge. This is also the case with Case 3 if we focus on the following voting situation on $A=\{a, b, c\}$ with 5 voters:

$$
3: a b c \quad 2: c a b
$$

Candidate $a$ is the Condorcet winner and candidate $c$ the Condorcet loser: $(a, b)$ is selected by $\mathcal{S}$. The reader can check that $a c b$ is the collective ranking with $\mathcal{V}$. Thus, give that there is a Condorcet winner and a Condorcet loser, $\mathcal{S}$ and $\mathcal{V}$ may diverge.

\section{D-Few words on the Verdoolaege et al. (2004)'s methodology}

The parameterized Barvinok's algorithm of Verdoolaege et al. (2004) is one of the methods for computing Ehrhart polynomials. It covers the limitations of the algorithm of Clauss and Loechner (1998) and it extends the Barvinok (1994)'s algorithm by considering parametric polytopes with any numbers of parameters.

The Barvinok's algorithm is helpful in the polynomial-time counting of integer points inside rational polyhedra of fixed dimension. Given a rational polyhedron $P$, the Barvinok's algorithm first defines a generating function $f(P, x)=\sum_{\eta \in P \cap \mathbb{Z}^{d}} x^{\eta}$ where $x=\left(x_{1}, x_{2}, \ldots, x_{d}\right), \eta=\left(\eta_{1}, \eta_{2}, \ldots, \eta_{d}\right)$

and $x^{\eta}=\prod_{i=1}^{d} x_{i}^{\eta_{i}}$. The parameter $\eta$ runs over all the integer lattice points included in $P \cap \mathbb{Z}^{d}$. By fixing $x_{i}=1$ for $1 \leq i \leq d, f(P, x)$ returns the number of integer lattice points contained in $P$. 
According to Brion (1998), $f(P, x)$ is equal to the sum over all the vertices $v$ of the generating functions of the supporting cones of $P$ at each vertex $v$. A supporting cone at a vertex is the polyhedron defined by the constraints that are saturated by the vertex. The cones are decomposed into unimodular cones ${ }^{17}$. Then, the generating functions of these cones which are rational functions are obtained easily and are simple to handle. It comes that $f(P, x)$ is the sum these rational functions. Going from $x=(1,1, \ldots, 1)$, the evaluation of $f(P, x)$ at this point is obtained by computing the residues.

The Parameterized Barvinok's algorithm of generalizes the Barvinok's algorithm by considering validity domains with the ability to handle periodic numbers. For more details on the , the reader can refer to the paper of Verdoolaege et al. (2004).

Acknowledgements. The author is grateful to Vincent Merlin and to anonymous reviewers for all their useful comments. Thanks to all the participants of the voting session at the 2013 Annual Meeting of the Public Choice Society in New Orleans (USA).

\section{References}

Barberà S., Coelho D. (2008) How to choose a non-controversial list with k names. Social Choice and Welfare 31: 79-96.

Bartholdi J., Tovey C.A. and Trick M.A. (1989) Voting schemes for which it can be difficult to tell who won the election. Social Choice and Welfare 6(3): 157-165.

Betzler N., Guo J. and Niedermeier R. (2008) Parameterized computational complexity of Dodgson and Young elections. In Algorithm Theory - SWAT 2008.

Barvinok A. (1994) Polynomial time algorithm for counting integral points in polyhedra when the dimension is fixed. Mathematics of Operations Research 19: 769-779.

Barvinok A., Pommersheim J. (1999) An algorithmic theory of lattice points in polyhedra. In: New Perspectives in Algebraic Combinatorics, Berkeley, CA, 1996-1997. Math. Sci. Res. Inst. Publ. 38: $91-147$.

Brion M. (1998) Points entiers dans les polyhèdres convexes. Annales Scientifiques de l'Ecole Normale Supérieure 21 (4): 653-663.

Bruynooghe M., Cools R., Verdoolaege S. and Woods K. (2005) Computation and manipulation of enumerators of integer projections of parametric polytopes. Technical Report CW 392. Katholieke Universiteit Leuven, Department of Computer Sciences.

Caragiannis I., Covey J., Feldman M., Christopher M. Homan C., Kaklamanis C., Karanikolas N., Procaccia A.D and Rosenscheiny J.S (2012) On the Approximability of Dodgson and Young Elections. Artificial Intelligence 187-188: 31-51.

Clauss P., Loechner V. (1998) Parametric analysis of polyhedral iteration spaces. Journal of VLSI Signal Processing 2(19): 179-194.

\footnotetext{
${ }^{17} \mathrm{~A}$ cone unimodular if its generators form a basis of the lattice $\mathbb{Z}^{d}$.
} 
Coelho D (2004) Understanding, evaluating and selecting voting rules through games and axioms. Phd Thesis, Universitat Autonoma de Barcelona.

Marquis de Condorcet (1785) Essai sur l'Application de l'Analyse à la Probabilité des Décisions Rendues à la Pluralité des Voix. Paris.

Courtin S., Mbih B., Moyouwou I. (2012) Are Condorcet procedures so bad according to the reinforcement axiom? Thema Working paper no 2012-37, Cergy Pontoise.

Dodgson C.L. (1876) A Method of Taking Votes on More than Two Issues. Clarendon Press, Oxford.

Ehrhart E. (1962). Sur les polyèdres rationnels homothétiques à $n$ dimensions. Comptes Rendus de l'Academie des Sciences Paris 254 : 616-618.

Ehrhart E. (1967) Sur un problème de géométrie diophantienne linéaire. Ph.D. Thesis. Journal für die Reine und Angewandte Mathematik. 226: 1-49.

Fishburn P.C. (1977) Condorcet Social Choice Functions. SIAM Journal on Applied Mathematics 33: 469-489.

Gehrlein W.V., Fishburn P.C. (1976) The probability of the paradox of voting: A computable solution. Journal of Economic Theory 13: 14-25.

Gehrlein W.V. (1985) The Condorcet criterion and committee selection. Mathematical Social Sciences 10: 199-209.

Gehrlein W.V., Lepelley D. (2010) Voting Paradoxes and Group Coherence. Springer.

Haung H.C, Chua V.C. (2000) Analytical representation of probabilities under IAC condition. Social Choice and Welfare 17: 143-155.

Hemaspaandra E., Hemaspaandra L. and Rothe J. (1997) Exact analysis of Dodgson elections: Lewis Carroll's 1876 voting system is complete for parallel access to NP, Journal of the ACM 44(6): 806-825.

Kamwa E. (2013) The Kemeny rule and committee elections. Economics Bulletin 33(1):648-654.

Kamwa E., Merlin V. (2013) Coincidence of Condorcet committees. Submitted paper, Mimeo, Normandy University.

Kamwa E. (2015a) On the Fishburn's social choice function. International Journal of Economic Theory 11(2).

Kamwa E. (2015b) On stable voting rules for electing committees. Submitted paper, Mimeo, Normandy University.

Kemeny J. (1959) Mathematics without numbers. Daedalus 88:571-591.

Kemeny J., Snell I. (1960) Mathematical Models in the Social Sciences. Boston: Ginn.

Kramer G.H. (1977) A dynamical model of political equilibrium. Journal of Economic Theory 16: 310-334. 
Lepelley D., Louichi A., Smaoui H. (2008) On Ehrhart polynomials and probability calculations in voting theory. Social Choice and Welfare 30(3):363-383.

Miller N.R. (1980) A new solution set for tournaments and majority voting : Further graphtheoretical approaches to the theory of voting. American Journal of Political Science 21 :68-96, 1980 .

Ratliff T.C. (2003) Some startling inconsistencies when electing committees. Social Choice and Welfare 21: 433-454.

Schwartz T. (1986) The Logic of Collective Choice. New York: Columbia University Press.

Simpson P. (1969) On defining areas of voter choice. Quarterly Journal of Economics 83: 478-490.

Verdoolaege S., Seghir R., Beyls K., Loechner V. and Bruynooghe M. (2004) Analytical computation of Ehrhart polynomials: enabling more compiler analysis and optimizations, in: Proceedings of International Conference on Compilers, Architecture and Synthesis for Embedded Systems, Washington DC.

Young H.P. (1975) Social Choice scoring functions. SIAM Journal of Applied Mathematics 28: 824-838.

Young H.P. (1977) Extending Condorcet's rule. Journal of Economic Theory 16:335-353.

Young H.P and Levenglick A.(1978) A consistent extension of Condorcet's election principle. SIAM Journal of Applied Mathematics $35: 285-300$. 